\title{
The association between body mass index and outcomes in patients with sepsis and acute respiratory failure
}

\author{
Edna Juarez MD, Hawa Edriss MD, Michelle Lear BS, Asley Sanchez BS, Shengping Yang PhD, Kenneth Nugent MD
}

\begin{abstract}
Background: Patients with increased body mass indices (BMIs) often present practical problems during their management for acute medical disorders in intensive care units. Some studies suggested obese patients have longer ICU stays but lower in-hospital mortality rates. This study considers the effect of BMI on outcomes in patients with sepsis and respiratory failure.

Methods: The electronic medical records of patients hospitalized between 2010 and 2016 with sepsis who required mechanical ventilation were reviewed to collect demographic characteristics, clinical information including BMI subcategory, management requirements, and outcomes, such as mortality, ICU length of stay (LOS), and hospital LOS.

Results: This study included 312 adult patients. The mean age was $59.1 \pm 16.3$ years; $57.4 \%$ were men. The mean BMI was $29.3 \pm 10.7 \mathrm{~kg} / \mathrm{m}^{2}$. The median APACHE II score was $14,46.2 \%$ of the patients had pulmonary infections, and $34.9 \%$ had extrapulmonary infections. The overall mortality was $42.6 \%$. The mean LOS was $12.4 \pm 11.8$ days in the ICU and $16.6 \pm 13.6$ days in the hospital. The mortality rates were $38.5 \%$ in underweight patients (BMI $\left.<18.5 \mathrm{~kg} / \mathrm{m}^{2}\right), 51.1 \%$ in normal weight patients (BMI $\left.18.5-24.9 \mathrm{~kg} / \mathrm{m}^{2}\right), 38.5 \%$ in overweight patients (BMI 25-29.9 kg/m ${ }^{2}$ ), and $40.2 \%$ in obese patients $\left(B M I>30 \mathrm{~kg} / \mathrm{m}^{2}\right)$. Body mass index did not have an independent effect on mortality after adjusting for age, gender, and APACHE II scores; however, overweight patients required more mechanical ventilation days and had an increased LOS in the ICU and the hospital.

Conclusions: This study demonstrates that patients with sepsis requiring mechanical ventilation have a high mortality rate and that BMI did not have an effect on mortality. A prospective study which considers differences in clinical characteristics, management requirements, complications, and inflammatory parameters in different BMI subcategories is needed. Overweight patients had an increased ICU and hospital LOS.
\end{abstract}

Keywords: body mass index, obesity, sepsis, acute respiratory failure, outcomes

\section{INTRODUCTION}

Obesity is a complex, multifactorial disease that has become one of the greatest challenges for public health around the globe, with an increasing

Corresponding author: Kenneth Nugent Contact Information: Kenneth.nugent@ttuhsc.edu DOI: $10.12746 /$ swrccc.v7i31.615 prevalence in adults and children. Obesity is defined by body mass indices (BMls in $\mathrm{kg} / \mathrm{m}^{2}$ ), hip to waist ratios, and the percentage of body fat. In general, overweight and obese patients have greater morbidity and mortality from many acute and chronic diseases, including acute coronary syndrome, gout, arthritis, biliary disease, diabetes, hypertension, and some cancers with an estimated excess mortality in the United States of 300,000 adults every year. ${ }^{1-3}$ Obesity increases health care system costs and is estimated 
to account for $5.7 \%$ of United States national health expenditures or approximately $\$ 190$ billion per year. ${ }^{1-3}$ Globally, the prevalence of overweight BMIs and obese BMls increased by $28 \%$ in adults and $47 \%$ in children between 1980 and 2013, and an estimated 2.1 billion people worldwide are either overweight or obese in 2016. ${ }^{4}$ Thirty-five percent of United States adult population is obese according to the National Health and Nutrition Examination Survey of $2016 .{ }^{5}$

Obesity has been considered a risk factor for sepsis and the development of septic shock. For example, Wang et al reported that a BMI $>40 \mathrm{~kg} / \mathrm{m}^{2}$ increased the risk of sepsis events (hazard ratio [HR]: 1.57; 95\% confidence interval $[\mathrm{Cl}]: 1.16-2.14)$ in their population based study of over thirty thousand patients. ${ }^{6}$ Twig et al analyzed data on 2.3 million adolescents and demonstrated a strong association between overweight and obesity and infectious disease mortality, especially bacterial infection, with HRs of $1.9(95 \%$ $\mathrm{Cl}: 1.4-2.5)$ for overweight adolescents and 2.5 (95\% $\mathrm{Cl}: 1.5-4.2)$ for obese adolescents. ${ }^{7}$ Patients with these clinical syndromes often require intensive care management.

Multiple factors, including the primary diagnosis, comorbidity, the level of acute stress, management strategies, and complications, affect outcomes in critically ill patients. Several studies have studied the relationship between obesity and mortality in critically ill patients, but the results from these studies are inconsistent. ${ }^{8}$ Zhao et al analyzed the association between $\mathrm{BMI}$ and outcomes in mechanically ventilated adult patients in intensive care units. ${ }^{9}$ They found that a higher BMI was associated with lower mortality rates and a longer duration mechanical ventilation but did not classify the patients based on the underlying diagnoses or indications for mechanical ventilation. Our study objective was to evaluate the effect of BMI on mortality in patients with sepsis and acute respiratory failure requiring mechanical ventilation for more than 24 hours. This cohort is an important group of patients in ICUs because of the high mortality rate associated with sepsis and mechanical ventilation and the increasing frequency of obese patients admitted to ICUs.

\section{METHODS}

We conducted an Institutional Review Board (IRB) approved retrospective review of existing data for patients admitted with diagnosis of severe sepsis or septic shock to the medical intensive care unit (MICU) at University Medical Center in Lubbock, Texas, between March 1,2010 to March 31, 2016. The charts were initially identified by discharge codes (ICD codes 995.91 and 785.52) and then reviewed to determine whether or not the patients met study criteria. The diagnosis was based on the Third International Consensus Definitions for Sepsis and Septic Shock. ${ }^{10}$ We included adults 18 years or older, intubated for a minimum of 24 hours, and admitted into the MICU with the diagnosis of sepsis or septic shock. We excluded all patients younger than 18 years and older than 89 years, all other types of shock, and patients requiring mechanical ventilation for less than 24 hours. Our IRB restricts retrospective studies to patients less than or equal to 89 years, and all information was deidentified.

We extracted the following information from the electronic medical records (EMRs): gender, age, BMI based on the admission weight and height, initial vital signs (either in the emergency department or the MICU), the first available laboratory tests (hemoglobin, white blood cell counts, lactate, BUN, creatinine, albumin, $\mathrm{pH}, \mathrm{PaCO}_{2}$, bicarbonate), APACHE II score on admission to the MICU, source of sepsis (respiratory, gastrointestinal, urinary, skin and subcutaneous tissue, neurologic, bloodstream, bone, endocarditis, mixed), in-hospital mortality, ICU length of stay (LOS), hospital LOS, length of mechanical ventilation (MV), comorbidities (limited to diabetes, end stage renal disease [ESRD], chronic obstructive pulmonary disease [COPD], chronic heart failure [CHF], obesity hypoventilation syndrome [OHS], and liver disease), initial chest x-ray results (clear lung fields, pleural effusion, focal or bilateral infiltrates, pneumothorax), renal replacement therapy during MICU management, and vasopressor support.

We used the following classification for BMI: BMI $<18.49 \mathrm{~kg} / \mathrm{m}^{2}$, underweight; BMI 18.5 to $24.9 \mathrm{~kg} / \mathrm{m}^{2}$, normal weight; BMI 25 to $29.9 \mathrm{~kg} / \mathrm{m}^{2}$, overweight; BMI 
30 to $34.9 \mathrm{~kg} / \mathrm{m}^{2}$, obese class 1 ; BMI 35 to $39.9 \mathrm{~kg} / \mathrm{m}^{2}$, obese class 2; BMI $>40 \mathrm{~kg} / \mathrm{m}^{2}$, obese class 3 .

Physicians in the Department of Internal Medicine at Texas Tech University Health Sciences Center manage patients admitted to the medical intensive care unit at University Medical Center who require mechanical ventilation. This management either involves direct care or consultative care. The Department of Internal Medicine has a pulmonary and critical care fellowship, and these trainees are involved in the management of these patients. University Medical Center has a code sepsis protocol designed to identify patients with possible sepsis as early as possible and to complete critical management steps, including cultures, the initiation of antibiotic therapy, and fluid administration, as soon as possible. Patients identified by the code sepsis protocol are routinely reviewed at 6 hours to make certain the sepsis bundle has been completed. Indications for mechanical ventilation included acute respiratory failure, altered mental status with inability to protect the airway, and refractory shock.

We hypothesized that an increased BMI would reduce mortality rate in patients with severe sepsis or septic shock requiring mechanical ventilation. Therefore, the primary outcome in this study was in-hospital mortality; secondary outcomes were the duration of mechanical ventilation, ICU LOS, and hospital LOS. We assumed that patients with normal BMls would have a $50 \%$ in-hospital mortality and that overweight and obese patients would have a lower rate (odds ratio of 0.4 ). Also, assuming that the study cohort had approximately equal numbers of normal BMI and overweight and obese patients, then, at a 0.05 significance level, in order to achieve $80 \%$ power, a total of 167 patients would be required to detect such a difference using a logistic regression.

Descriptive statistics were used to describe the characteristics of the study cohort. Categorical variables were summarized as frequencies, and continuous variables were summarized using means and standard deviations or medians and ranges, as appropriate. Univariate logistic regression was used to test if there was a significant association between mortality and BMI on admission. Multiple logistic regression was used to test such associations while adjusting for other risk factors, including age, gender, APACHE II scores, the number of comorbid conditions, and a requirement for acute renal replacement therapy, which are potentially associated with mortality. Odds ratios (ORs) and their 95\% confidence intervals (Cls) were calculated to evaluate these associations. A sensitivity analysis using albumin as a continuous variable was performed on mortality and length of stay calculations to determine the effect of nutrition on outcomes. Poisson regression was used to assess the association between BMI and ICU and hospital LOS, while adjusting for all risk factors. The reference group for these analyses was the patients with a normal BMI $\left(18.5\right.$ to $\left.24.9 \mathrm{~kg} / \mathrm{m}^{2}\right)$. Statistical significant level was set at 0.05 . Multiple testing adjustment was not performed. Analyses were performed using SAS software (Windows version 9.3; SAS Institute, Cary, NC).

This study had approval by the Texas Tech University Health Science Center Institutional Review Board in Lubbock, Texas.

\section{RESULTS}

We reviewed 336 EMRs of patients admitted into the MICU between 2010 and 2016. Of these EMRs, twenty-four where excluded, including five due to a non-infectious etiology of shock and eighteen due to mechanical ventilation for less than 24 hours. One patient was excluded because respiratory support was with noninvasive ventilation.

Of the 312 EMRs included in the final cohort, 179 were males $(57.4 \%)$, and 133 were females (42.6\%) (Table 1). The mean age was $59.1 \pm 16.3$ years; the mean BMI was $29.3 \pm 10.7 \mathrm{~kg} / \mathrm{m}^{2}$. The underweight group had 26 patients $(8.3 \%)$, the normal BMI group had 88 patients $(28.2 \%)$, the overweight group had 91 patients $(29.2 \%)$, and the obese class I group had 36 patients $(11.5 \%)$, the obese class II group had 32 patients $(10.3 \%)$, and the obese class III group had 39 patients $(12.5 \%)$. The median number of comorbidities for all patients was 1; the obese class III group had a median of two comorbidities. Sources of infection included pulmonary $(46.2 \%)$, extra pulmonary $(34.9 \%)$, and mixed infection $(18.9 \%)$ and were 


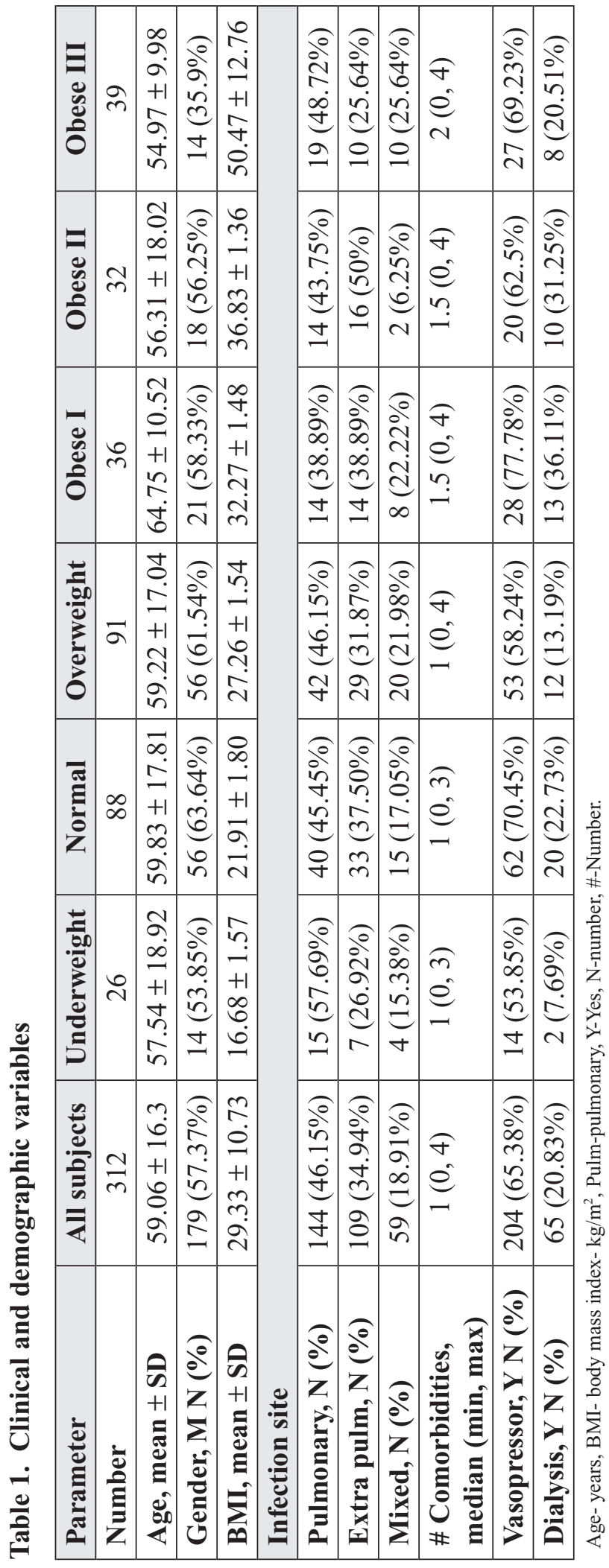

similar in all BMI categories (Table 1). Two hundred and four patients $(65.4 \%)$ required vasopressors during their admission, and $65(20.8 \%)$ required acute renal replacement therapy. The obese class I and II groups had a slightly higher percentage of subjects requiring acute renal replacement therapy with $36.1 \%$ and $31.3 \%$, respectively, and the underweight group had a lower percentage of subjects requiring acute renal replacement therapy with $7.7 \%$ (Table 1 ).

The mean creatinine on admission was $2.0 \mathrm{mg} / \mathrm{dl}$; the obese class I and II patients had a higher mean initial creatinine than patients in other BMI categories (Table 2). The mean albumin levels were $3.2 \mathrm{~g} / \mathrm{dl}$, with lower levels in the underweight group and higher levels in the normal BMI and obese class I groups. The median APACHE II score was 14 (3-33), with the highest median APACHE II score in the normal BMI group (16) and the lowest in the underweight and obese groups (13.5-14). Other clinical and laboratory variables are reported in Table 2.

The highest mortality was in the normal BMI group $(51.1 \%)$, and the lowest mortality rate was in the underweight group (38.5\%) and overweight group $(38.5 \%)$. Table 3 reports the analysis of the association between mortality and BMI adjusted for other risk factors. The mortality was lower in the underweight, overweight, and obese groups, but this was not statistically significant after adjustment for other factors. Age was positively associated with mortality (adjusted OR 1.03; 95\% Cl 1.01-1.05), and mortality was higher in patients requiring dialysis (OR 2.26; 95\% Cl 1.17-4.46) (Table 3). The APACHE II score was positively associated with mortality in an unadjusted analysis (unadjusted OR:1.04; 95\% Cl 1.00$1.08)$ but not after adjustment for other factors (OR: $1.01 ; 95 \%$ Cl 0.96-1.05).

The duration of mechanical ventilation, ICU LOS, and hospital LOS are reported in Table 4. Underweight patients had a shorter period of mechanical ventilation and ICU LOS. Overweight patients had a longer ICU LOS. Factors associated with mechanical ventilation time, ICU LOS, and hospital LOS are reported in Table 5. Mechanical ventilation was longer in men than in women $(P=0.034)$. The underweight patients had the shortest ICU LOS $(P=0.028)$ compared to the 


\begin{tabular}{|c|c|c|c|c|c|c|c|c|c|c|c|c|}
\hline $\begin{array}{l}\text { 三 } \\
0 \\
0 \\
0 \\
0 \\
0\end{array}$ & बे & $\begin{array}{l}+ \\
\infty \\
i \\
0 \\
+1 \\
a \\
\vdots \\
\hat{a}\end{array}$ & 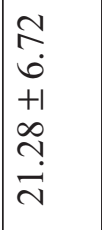 & 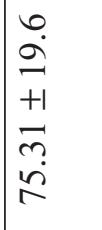 & $\begin{array}{l}a \\
i \\
i \\
+1 \\
\infty \\
n \\
\vdots \\
a\end{array}$ & 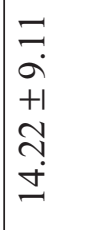 & $\begin{array}{l}\infty \\
n \\
i \\
+1 \\
+ \\
\tilde{n} \\
=\end{array}$ & $\begin{array}{l}\infty \\
\vec{i} \\
+1 \\
+1 \\
0 \\
\vdots \\
-\end{array}$ & 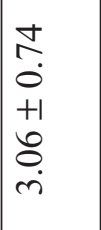 & $\begin{array}{l}\text { ते } \\
\text { in } \\
+1 \\
\text { ते } \\
\text { ì }\end{array}$ & $\begin{array}{l}\tilde{n} \\
m \\
n \\
n \\
m \\
n\end{array}$ & 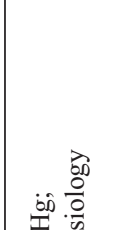 \\
\hline $\begin{array}{l}\exists \\
0 \\
: \\
0 \\
0\end{array}$ & 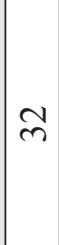 & $\begin{array}{l}\sigma \\
0 \\
\infty \\
N \\
+1 \\
\infty \\
\infty \\
\dot{0} \\
0\end{array}$ & $\begin{array}{l}7 \\
6 \\
+1 \\
0 \\
0 \\
\dot{\imath}\end{array}$ & $\begin{array}{l}\tilde{N} \\
\stackrel{\sim}{+} \\
+1 \\
\sigma \\
\dot{\infty}\end{array}$ & \begin{tabular}{l}
$\underset{8}{0}$ \\
$\dot{a}$ \\
+1 \\
\multirow{2}{*}{} \\
$\infty$ \\
$\infty$ \\
$a$
\end{tabular} & 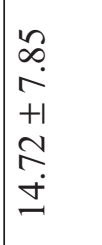 & 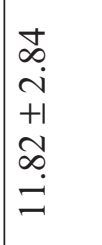 & $\begin{array}{l}\text { i } \\
i \\
i \\
+1 \\
b \\
i\end{array}$ & 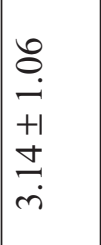 & \begin{tabular}{l}
$n$ \\
0 \\
0 \\
+1 \\
+1 \\
$\sim$ \\
\hdashline \\
$\dot{n}$
\end{tabular} & $\begin{array}{l}\text { ปे } \\
\dot{ \pm} \\
\pm\end{array}$ & 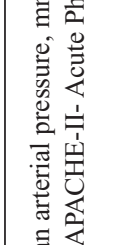 \\
\hline $\begin{array}{l}\overrightarrow{0} \\
0 \\
0 \\
0 \\
0\end{array}$ & \% & $\begin{array}{l}2 \\
2 \\
2 \\
+1 \\
\infty \\
0 \\
0 \\
0 \\
0\end{array}$ & $\begin{array}{l}m \\
0 \\
\infty \\
+1 \\
0 \\
0 \\
n \\
\end{array}$ & $\begin{array}{l}n \\
\tilde{a} \\
\dot{N} \\
+1 \\
n \\
\dot{\infty}\end{array}$ & \begin{tabular}{l}
0 \\
$b$ \\
\hdashline+1 \\
+1 \\
+ \\
$m$ \\
$a$ \\
$a$
\end{tabular} & $\begin{array}{l}2 \\
i \\
i \\
+1 \\
\sim \\
n \\
n \\
n\end{array}$ & $\begin{array}{l}n \\
n \\
i \\
+1 \\
+ \\
n \\
= \\
=\end{array}$ & $\begin{array}{l}\stackrel{m}{a} \\
+1 \\
i \\
i \\
i\end{array}$ & 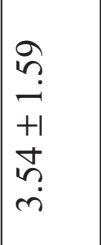 & $\begin{array}{l}\infty \\
i \\
+1 \\
\infty \\
\infty \\
\dot{n}\end{array}$ & 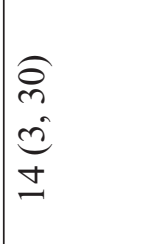 & 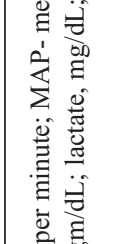 \\
\hline 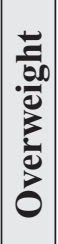 & $\bar{a}$ & 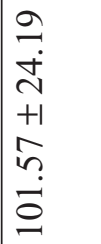 & 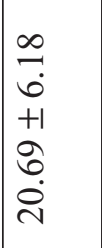 & $\begin{array}{l}2 \\
\dot{0} \\
\hat{\lambda} \\
+1 \\
m \\
0 \\
\infty\end{array}$ & \begin{tabular}{l}
$\vec{a}$ \\
\hdashline+1 \\
$a$ \\
$a$ \\
$\infty$
\end{tabular} & $\begin{array}{l}\hat{2} \\
i \\
+1 \\
0 \\
0 \\
\dot{I}\end{array}$ & $\begin{array}{l}\vec{N} \\
i n \\
+1 \\
0 \\
\vec{d} \\
\end{array}$ & $\begin{array}{l}n \\
i \\
+1 \\
\infty \\
\infty \\
a \\
-1\end{array}$ & $\begin{array}{l}\vec{\infty} \\
0 \\
+1 \\
+1 \\
0 \\
\dot{m}\end{array}$ & $\begin{array}{l}\sigma \\
\hat{i} \\
+1 \\
\tilde{0} \\
\dot{n}\end{array}$ & 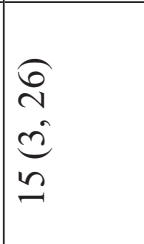 & 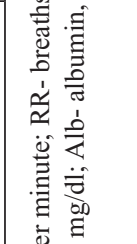 \\
\hline 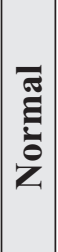 & $\infty$ & 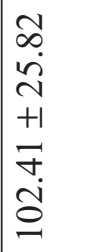 & $\begin{array}{l}\hat{n} \\
\hat{i} \\
+1 \\
\hat{\alpha} \\
\hat{i}\end{array}$ & $\begin{array}{l}n \\
0 \\
2 \\
+1 \\
+1 \\
i \\
b \\
0 \\
2\end{array}$ & $\begin{array}{l}\text { fr } \\
i \\
+1 \\
o \\
\infty \\
\vdots \\
\vdots \\
a\end{array}$ & $\begin{array}{l}\vec{n} \\
a \\
+1 \\
m \\
\dot{J}\end{array}$ & $\begin{array}{l}0 \\
\dot{0} \\
\dot{n} \\
+1 \\
n \\
n \\
=\end{array}$ & 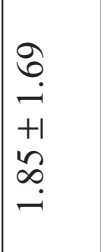 & $\begin{array}{l}2 \\
\partial \\
0 \\
+1 \\
\dot{n} \\
\tilde{n} \\
\tilde{n}\end{array}$ & $\begin{array}{l}2 \\
\infty \\
\dot{n} \\
+1 \\
\hat{a} \\
\dot{n}\end{array}$ & $\begin{array}{l}\tilde{n} \\
\tilde{n} \\
0 \\
0 \\
0\end{array}$ & 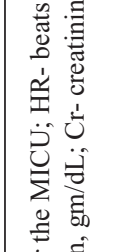 \\
\hline 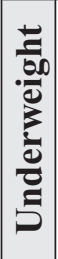 & 눈 & $\begin{array}{l}\infty \\
n \\
\\
+1 \\
\infty \\
0 \\
\infty \\
0 \\
0\end{array}$ & 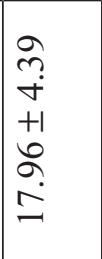 & 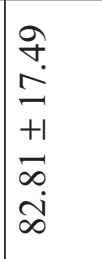 & $\begin{array}{l}0 \\
i \\
i \\
+1 \\
n \\
n \\
a \\
a\end{array}$ & $\begin{array}{l}\text { O } \\
\infty \\
0 \\
+1 \\
+1 \\
\text { i } \\
\text { i }\end{array}$ & $\begin{array}{l}0 \\
n \\
n \\
+1 \\
0 \\
0 \\
0 \\
0 \\
1\end{array}$ & $\begin{array}{l}2 \\
a \\
0 \\
+1 \\
n \\
n \\
-1\end{array}$ & $\begin{array}{l}\hat{n} \\
0 \\
+1 \\
\hat{a} \\
i \\
i\end{array}$ & $\begin{array}{l}J \\
\dot{j} \\
+1 \\
a \\
a \\
i\end{array}$ & $\begin{array}{l}\sqrt{2} \\
\pm \\
\pm\end{array}$ & 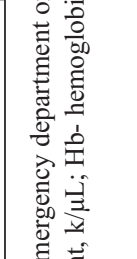 \\
\hline 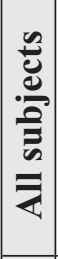 & $\stackrel{2}{m}$ & $\begin{array}{l}n \\
n \\
n \\
n \\
+1 \\
n \\
0 \\
\dot{0} \\
0\end{array}$ & $\begin{array}{l}\text { N } \\
0 \\
0 \\
+1 \\
\infty \\
0 \\
0 \\
0\end{array}$ & $\begin{array}{l}1 \\
\alpha \\
\stackrel{N}{1} \\
+1 \\
\infty \\
2 \\
2\end{array}$ & $\begin{array}{l}2 \\
\vec{i} \\
+1 \\
0 \\
0 \\
0 \\
\vdots \\
a\end{array}$ & \begin{tabular}{l}
$\vec{F}$ \\
$\infty$ \\
+1 \\
+1 \\
\multirow{+}{+}{} \\
$\dot{I}$
\end{tabular} & $\begin{array}{l}\infty \\
\dot{n} \\
+1 \\
\dot{0} \\
\dot{\Xi}\end{array}$ & $\begin{array}{l}\sim \\
a \\
+1 \\
+1 \\
d \\
i\end{array}$ & $\begin{array}{c}2 \\
a \\
0 \\
+1 \\
0 \\
0 \\
\dot{n}\end{array}$ & $\begin{array}{l}0 \\
N \\
n \\
+1 \\
+1 \\
n \\
n \\
n\end{array}$ & $\begin{array}{l}\text { } \\
\tilde{n} \\
\infty \\
\pm\end{array}$ & 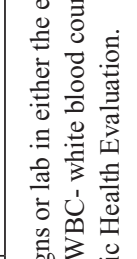 \\
\hline 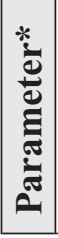 & 㐫 & 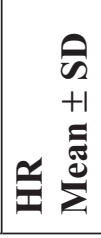 & 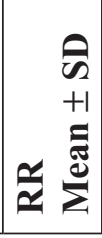 & 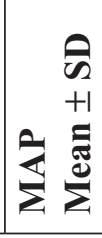 & 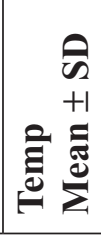 & 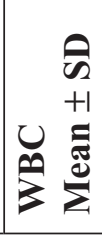 & 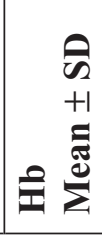 & 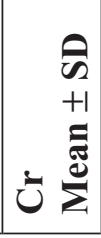 & 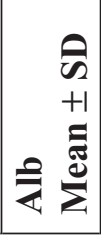 & 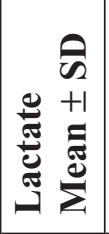 & 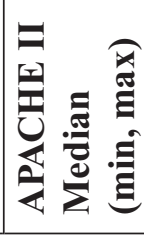 & 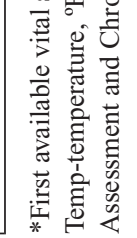 \\
\hline
\end{tabular}


Table 3. In hospital mortality and clinical variables

\begin{tabular}{|c|c|c|c|c|}
\hline & Alive & Dead & Odds Ratio (raw) & Odds Ratio (adjusted) \\
\hline $\mathbf{N}$ & $(\mathrm{n}=179)$ & $(n=133)$ & & \\
\hline Age, mean \pm SD & $56.6(16.3)$ & $62.4(15.8)$ & $1.02(1.01,1.04)$ & $1.03(1.01,1.05)$ \\
\hline \multicolumn{5}{|l|}{ Gender } \\
\hline Female, N (\%) & $80(44.69)$ & $53(39.85)$ & Reference & Reference \\
\hline Male, N (\%) & $99(55.31)$ & $80(60.15)$ & $1.22(0.77,1.92)$ & $1.42(0.84,2.41)$ \\
\hline \multicolumn{5}{|l|}{ BMI } \\
\hline Normal & $43 / 88(48.9)$ & $45 / 88(51.1)$ & Reference & Reference \\
\hline Underweight & $16 / 26(61.5)$ & $10 / 26(38.5)$ & $0.60(0.24,1.46)$ & $0.60(0.20,1.69)$ \\
\hline Overweight & $56 / 91(61.4)$ & $35 / 91(38.5)$ & $0.60(0.33,1.08)$ & $0.67(0.34,1.32)$ \\
\hline Obese I & $20 / 36(55.6)$ & $16 / 36(44.4)$ & $0.76(0.35,1.66)$ & $0.65(0.26,1.59)$ \\
\hline Obese II & $21 / 32(65.5)$ & $11 / 32(34.4)$ & $0.50(0.21,1.14)$ & $0.51(0.20,1.28)$ \\
\hline Obese III & $23 / 39(59.0)$ & $16 / 39(41.0)$ & $0.66(0.31,1.41)$ & $1.05(0.41,2.64)$ \\
\hline \# comorbidity (Min, Max) & $1.0(0.0,4.0)$ & $2.0(0.0,4.0)$ & $1.11(0.89,1.39)$ & $0.94(0.71,1.25)$ \\
\hline APACHE II & $14.0(3.0,33.0)$ & $15.0(4.0,33.0)$ & $1.04(1.00,1.08)$ & $1.01(0.96,1.05)$ \\
\hline \multicolumn{5}{|l|}{ Dialysis } \\
\hline No & $150(84.27)$ & 95 (71.97) & & \\
\hline Yes & $28(15.73)$ & $37(28.03)$ & $2.09(1.20,3.63)$ & $2.26(1.17,4.46)$ \\
\hline
\end{tabular}

We compared the difference between including and without including albumin in the model, None of the significance levels changed.

normal BMI reference group. The overweight patients had a longer ICU LOS $(P=0.032)$. The obese class I patients had longer ICU LOS $(P=0.009)$ and hospital LOS $(P=0.005)$. The obese class II patients had a shorter hospital LOS $(P=0.024)$.
The patients were divided into 2 age cohorts, age $<60$ years old and age $\geq 60$ years old (Table 6 ). BMI category had no statistically significant effect on MV days, ICU LOS, hospital LOS, or mortality in patients less than 60 years of age. In patients older than

Table 4. Length of mechanical ventilation, ICU, and hospital stay per BMI group

\begin{tabular}{|l|c|c|c|c|c|c|}
\hline \multirow{2}{*}{ BMI } & \multicolumn{2}{|c|}{ MV days } & \multicolumn{2}{c|}{ LOS, ICU } & \multicolumn{2}{c|}{ LOS, Hospital } \\
\cline { 2 - 7 } & Mean \pm SD & P value & Mean \pm SD & P value & Mean \pm SD & P value \\
\hline Normal & $8.08 \pm 4.97$ & Reference & $10.30 \pm 8.02$ & Reference & $14.98 \pm 10.95$ & Reference \\
\hline Underweight & $5.48 \pm 3.25$ & $\mathbf{0 . 0 2 2}$ & $6.08 \pm 4.09$ & $\mathbf{0 . 0 0 3}$ & $13.81 \pm 11.08$ & 0.633 \\
\hline Overweight & $10.22 \pm 10.99$ & 0.100 & $13.30 \pm 13.09$ & $\mathbf{0 . 0 2 5}$ & $18.63 \pm 15.40$ & 0.054 \\
\hline Obese I & $8.85 \pm 5.61$ & 0.480 & $14.75 \pm 14.97$ & 0.079 & $22.56 \pm 23.05$ & 0.072 \\
\hline Obese II & $8.28 \pm 6.83$ & 0.964 & $9.48 \pm 7.55$ & 0.723 & $12.62 \pm 9.99$ & 0.293 \\
\hline Obese III & $8.5 \pm 5.43$ & 0.828 & $9.59 \pm 7.24$ & 0.935 & $14.23 \pm 13$ & 0.570 \\
\hline
\end{tabular}

Numbers in bold are statistically significant. 
Table 5. Factors associated with MV time, ICU and hospital length of stay

\begin{tabular}{|c|c|c|c|c|c|c|}
\hline & \multicolumn{2}{|c|}{ MV time } & \multicolumn{2}{|c|}{ ICU LOS } & \multicolumn{2}{|c|}{ Hospital LOS } \\
\hline & Estimate & P value & Estimate & $P$ value & Estimate & P value \\
\hline Age & -0.007 & 0.101 & -0.0026 & 0.4083 & -0.0054 & 0.080 \\
\hline \multicolumn{7}{|l|}{ Gender } \\
\hline Female & Reference & & Reference & & Reference & \\
\hline Male & 0.288 & 0.034 & 0.1437 & 0.1354 & 0.0813 & 0.384 \\
\hline \multicolumn{7}{|l|}{ BMI } \\
\hline Normal & Reference & & Reference & & Reference & \\
\hline Underweight & -0.463 & 0.083 & -0.4357 & 0.028 & 0.059 & 0.751 \\
\hline Overweight & 0.337 & 0.055 & 0.2638 & 0.032 & 0.220 & 0.067 \\
\hline Obese I & 0.167 & 0.480 & 0.4331 & 0.009 & 0.458 & 0.005 \\
\hline Obese II & -0.005 & 0.984 & -0.2272 & 0.181 & -0.371 & 0.024 \\
\hline Obese III & 0.235 & 0.342 & 0.0267 & 0.876 & -0.006 & 0.972 \\
\hline \# comorbidity & -0.116 & 0.114 & -0.0651 & 0.210 & -0.023 & 0.657 \\
\hline APACHE II & 0.011 & 0.343 & -0.0109 & 0.199 & -0.014 & 0.094 \\
\hline Dialysis & 0.256 & 0.138 & 0.349 & 0.004 & 0.220 & 0.065 \\
\hline
\end{tabular}

Numbers in bold are statistically significant. This analysis was repeated including albumin as a continuous variable. There was no change in the significance levels for MV time and ICU LOS.

60 years of age, mechanical ventilation, ICU LOS, and hospital LOS were increased in patients in the overweight and obese category 1 groups.

\section{Discussion}

Obesity is an independent risk factor for increased morbidity and mortality and represents the fifth leading cause of death in the world. ${ }^{3}$ The number of obese patients admitted into intensive care units has continued to increase in the United States; $30 \%$ of ICU patients are obese, and $7 \%$ are morbidly obese. ${ }^{2}$ Obesity seems to confer a higher risk of common conditions in critically ill patients, such as the acute respiratory distress syndrome (ARDS). ${ }^{11}$ Obesity might increase the morbidity and mortality of patients admitted to intensive care units, but this effect, if any, will depend on the admitting diagnosis, associated comorbidity, and the development of complications during intensive care management. In addition, obesity increases the incidence of some medical disorders, such as infections, which have high mortality rates.
However, studies on the effect of BMI on outcomes in critically ill patients have reported results which range from increased mortality to no effect on mortality to decreased mortality. Studies which suggest that obesity can reduce mortality in both acute and chronic illness have led to the idea of the obesity paradox. ${ }^{12,13}$ Our study did not find differences in the mortality in either overweight or obese patients with sepsis and acute respiratory failure requiring mechanical ventilation compared to patients with normal BMls. Also, patients with low BMls did not have increased mortality when compared to normal BMI patients. Age and the need for dialysis were associated with increased mortality in this patient cohort. Unadjusted APACHE II scores were associated with higher mortality (OR $1.04 ; 1.00,1.08)$, but this association was not significant after adjustment for other variables in our model (OR1.01; 0.96,1.05). In addition, sensitivity analysis using albumin concentrations as an indicator of nutrition as a continuous variable did not change outcomes in either the mortality model or the length of stay models. 


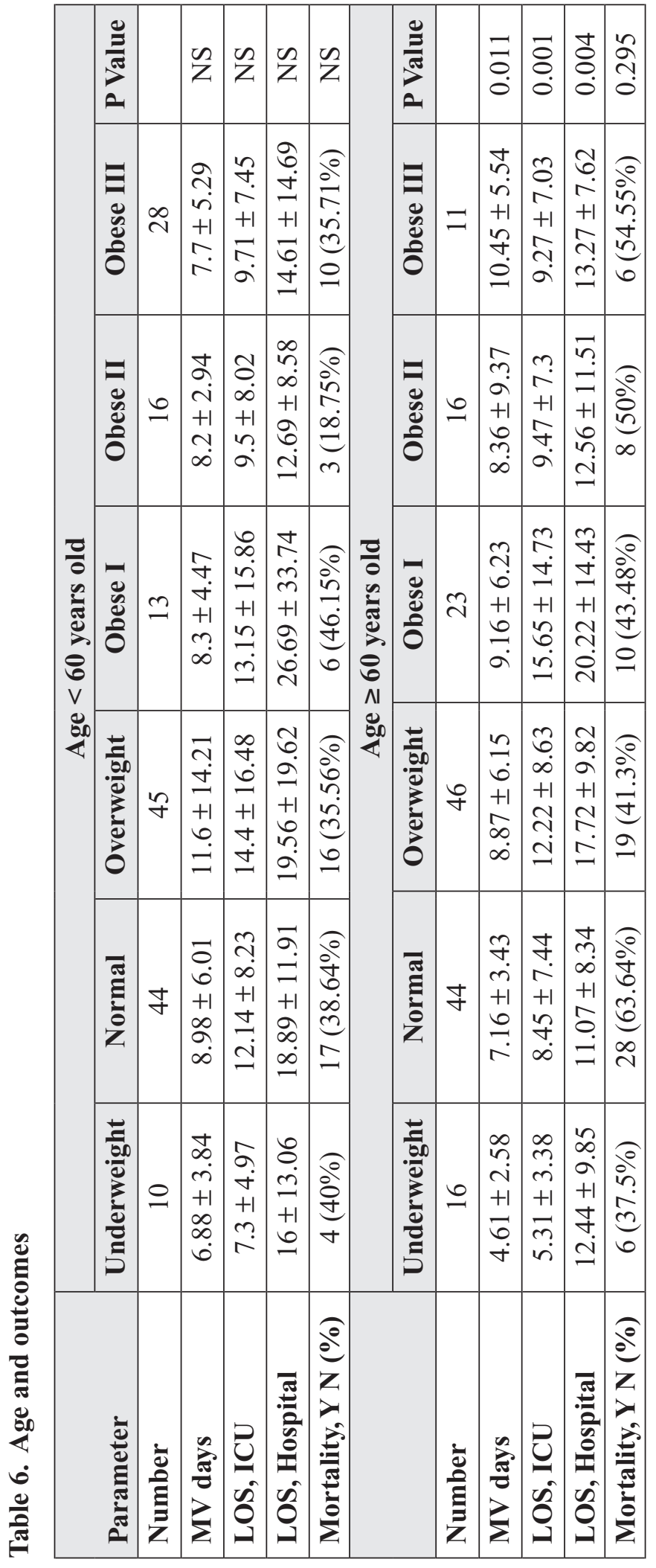

Several investigators have analyzed the association between obesity and outcomes in critically ill patients. Oliveros et al used a meta-analysis and systematic review to study the effect of obesity on mortality in critically ill adults. Obesity had a modest effect on outcomes. ${ }^{8}$ Overweight patients had decreased mortality, approximately $9 \%$, and obese patients had decreased mortality, approximately $18 \%$. Morbidly obese patients did not have a change in mortality. Obesity has a very slight effect on the length of stay, but these differences were less than a 1-day increase. This study did not specifically identify patients with sepsis or patients requiring mechanical ventilation. Pepper et al analyzed a large retrospective cohort with 55,038 patients from 139 hospitals in the United States and found that overweight and obese BMls reduced the adjusted odds ratio for mortality in ICU patients with sepsis or septic shock. ${ }^{14}$ This study did not adjust for interventions, such as mechanical ventilation or dialysis. Pepper also reported a meta-analysis which included data from four retrospective and two prospective studies (7,165 patients) and found a reduction in the adjusted OR (aOR) for all-cause mortality in overweight and obese patients (aOR: $0.83 ; 0.75,0.91 ; p<0.001 ; 0.82 ; 0.67,0.99 ; p=0.04$, respectively) admitted to the ICU with sepsis, severe sepsis, and septic shock. ${ }^{15}$ A morbidly obese BMI $\left(>40 \mathrm{~kg} / \mathrm{m}^{2}\right)$ based on three studies and an underweight BMI $\left(<18.5 \mathrm{~kg} / \mathrm{m}^{2}\right)$ based on three studies did not alter the aOR $(0.90 ; 0.59,1.39 ; p=0.64$; $1.24 ; 0.79,1.95 ; p=0.35$, respectively) for mortality. Consequently, the degree of obesity may influence outcomes in critically ill patients.

Wang et al reported a systematic review and meta-analysis on the effect of increased body mass indices on outcomes in patients with sepsis. ${ }^{16}$ They provided no information on the use of mechanical ventilation, dialysis, or vasopressors. Patients with a BMl greater than $25 \mathrm{~kg} / \mathrm{m}^{2}$ had decreased mortality, approximately $19 \%$. Overweight patients had decreased mortality, approximately 13\%. Obese patients and morbidly obese patients did not have decreased mortality. This study provides a significant amount of information about the frequency mortality and the length of stay. The percent mortality ranged from $6.3 \%$ to $52 \%$; it clearly depended on the subgroup 
and the study site(s). Length of stay ranged from 5 days to 34 days. This clearly depended on the study site(s) and the subgroup. Zhao et al also reported a systematic review and meta-analysis on the effect or association of body mass index on outcomes in mechanically ventilated adult patients in intensive care units. ${ }^{9}$ The underlying diagnosis requiring mechanical ventilation was not reported. They found that a higher BMI was associated with lower mortality and longer duration mechanical ventilation. Obese patients had a $12 \%$ reduction in mortality. Obese patients required mechanical ventilation for 0.48 days longer and an ICU length of stay for 0.38 days longer.

Tafelski prospectively studied postoperative patients who required more than 48 hours of ICU management. ${ }^{17}$ There was no difference in mortality between obese and non-obese patients. However, obese patients were more likely to require mechanical ventilation and were less likely to have therapeutic levels of vancomycin. This study suggested that antibiotic dosing in obese patients may be more difficult than in non-obese patients which could potentially influence outcomes in ICU related infections. A systematic review reported by Trivedi et al found no consistent effect of obesity on mortality associated with sepsis. ${ }^{18}$ This review included seven studies which reported no significant association with between obesity and mortality (3 studies), increased mortality associated with obesity (1 study), and decreased mortality associated with obesity (3 studies). In summary, obesity does not confer increased risk for mortality in critically ill patients requiring ICU care, including either patients with requiring mechanical ventilation or patients with sepsis, and may actually decrease the risk. These studies did not specifically analyze the effect of BMI on outcomes in patients with severe sepsis or septic shock who required mechanical ventilation.

\section{ANALYSIS BASED ON SPECIFIC DIAGNOSES}

Several studies have analyzed the effect of obesity on outcomes in critically ill patients with specific diagnoses. Soto reported that obesity was associated with the development of acute kidney injury in patients with the adult respiratory distress syndrome and that acute kidney injury was associated with increased mortality in a multivariable model. ${ }^{11}$ However, body mass index was associated with decreased mortality in this patient population using the same model. Danziger also studied the association between obesity and the development of acute kidney injury in critically ill patients. ${ }^{19}$ The odds of acute kidney injury increased in all BMI categories starting with the overweight group when compared to the normal BMI category. Patients with acute kidney injury had increased in the hospital and one-year mortality rates. Consequently, analyzing the effect of obesity on outcomes will depend on the identification of all clinical variables which have the potential to influence outcomes and their inclusion in multivariable models. Kok analyzed the association between obesity and outcomes and patients with cirrhosis and septic shock. ${ }^{20}$ This study included 362 patients and determined that mortality was increased with increased BMI, delays in the administration of appropriate antibiotics, and increased peak lactate levels. Bercault et al found increased mortality in 170 mechanically ventilated obese patients $\left(\mathrm{BMl}>30 \mathrm{~kg} / \mathrm{m}^{2}\right.$ ) when compared to matched patients with normal BMls with an OR 2.1 (95\% Cl: $1.3-3.5 ; p=0.003)$ using a risk adjusted analysis. ${ }^{1}$ This increased mortality was potentially explained by an increased frequency of complications during the ICU management. These last two studies demonstrate that management and the development of in-hospital complications can influence outcomes independent of the initial clinical diagnosis.

Malnutrition has been associated with increased mortality in patients admitted to general hospitals. Garrouste-Orgeas et al studied the outcomes in 1698 patients admitted into 6 medical-surgical ICUs. ${ }^{21}$ The overall hospital mortality in these patients was $31.3 \%$. In multivariate analysis patients with BMls $<18.5 \mathrm{~kg} / \mathrm{m}^{2}$ had an increased mortality rate (odds ratio 1.63; 1.11-2.39). Havens reported a retrospective study of patients undergoing emergency general surgery and admitted into the intensive care units following these surgical procedures and analyzed the effect of malnutrition identified by a registered dietitian's formal assessment on outcomes. ${ }^{22}$ This cohort included 1361 patients; $60 \%$ of these patients had nonspecific malnutrition. The 90-day mortality rate was $17.9 \%$. Patients with nonspecific malnutrition had an 
increased odds ratio for 90-day mortality (adjusted odds ratio $1.51 ; 1.09-5.04$ ). However, other studies have not found differences in mortality when comparing normal vs low BMI patients. ${ }^{23}$ A retrospective cohort study with 301 septic patients from Austria did not find increased mortality in patients with low BMls compared to patients with normal BMls. Aldawood analyzed outcomes in 1835 patients admitted to a tertiary care ICU. ${ }^{24}$ There is no difference in ICU mortality in various $\mathrm{BMI}$ groups, including low BMI patients. In our study, an abnormally low BMI was not associated with increased mortality but was associated with a statistically significant decrease in ICU LOS.

\section{LiMITATIONS}

This study was a retrospective single center study with a diverse patient population. Information was collected from electronic medical records and may have been incomplete. For example, the number of comorbidities could influence outcomes, and it is difficult to adjust the analysis based on the severity of any particular comorbidity in a given patient. Outcomes may reflect different management strategies by the teams involved in patient care. We did not investigate other parameters of malnutrition, such as prealbumin, and we did not correlate the BMI with body fat percentage or waist-hip ratios. We also did not analyze the time between the onset of symptoms and treatment that could have influenced the outcomes in these patients. However, our study only included patients with sepsis requiring mechanical ventilation which represents a somewhat uniform but complex patient population and had very clear outcomes. The high mortality rate in this study indicates that the diagnostic criteria selected a group of very sick patients, and these criteria may make it difficult to identify the contribution of other minor clinical factors to outcomes.

\section{Conclusions}

Our study demonstrates that patients with sepsis requiring mechanical ventilation have high mortality rates. Patients with increased BMls did not have increased or decreased mortality in comparison to patients in the normal reference range BMI in either unadjusted or adjusted analysis. Age and dialysis were associated with an increased the odds ratio for death in these septic patients with acute respiratory failure. Outcomes, such as ICU LOS and length of MV, were higher in overweight and some obese patients. Patients with low BMls did not have increased mortality rates, and, in fact, required less time on mechanical ventilators and had reduced ICU LOS. More prospective studies with well characterized patients are needed to establish the relationship between abnormal BMls and survival in critically ill patients.

Article citation: Juarez E, Edriss H, Lear M, Sanchez A, Yang S, Nugent K. The association between body mass index and outcomes in patient with sepsis and acute respiratory failure. The Southwest Respiratory and Critical Care Chronicles 2019;7(31):13-23.

From: Department of Internal Medicine (EJ, ML, AS, $\mathrm{KN})$, Texas Tech University Health Sciences Center, Lubbock, TX; Saint Joseph Hospital/Pulmonary Associates (HE), Lexington, KY; Pennington Biomedical Research Center (SY), Baton Rouge, LA

Submitted: 10/1/2019

Accepted:10/20/2019

Reviewer: Jeff Dennis PhD

Conflicts of interest: none

This work is licensed under a Creative Commons Attribution-ShareAlike 4.0 International License.

\section{REFERENCES}

1. Bercault N, Boulain T, Kuteifan K, Wolf M, Runge I, Fleury JC. Obesity-related excess mortality rate in an adult intensive care unit: A risk-adjusted matched cohort study. Critical Care Medicine 2004;32:998-1003.

2. Akinnusi ME, Pineda LA, El Solh AA. Effect of obesity on intensive care morbidity and mortality: A meta-analysis. Critical Care Medicine 2008;36:151-8.

3. Smith KB, Smith MS. Obesity Statistics. Primary Care: Clinics in Office Practice 2016;43:121-35.

4. www.who.int/en/news-room/fact-sheets/detail/obesityand-overweight

5. Hales CM, Carroll MD, Fryar CD, Ogden CL. Prevalence of obesity among adults and youth: United States, 2015-2016. NCHS Data Brief No. 288, October 2017. National Health 
and Nutrition Examination Survey. National Center for Health statistics 2017.

6. Wang HE, Griffin R, Judd S, Shapiro NI, Safford MM. Obesity and risk of sepsis: A population-based cohort study. Obesity 2013;21:E762-E9.

7. Twig G, Geva N, Levine H, et al. Body mass index and infectious disease mortality in midlife in a cohort of 2.3 million adolescents. International Journal of Obesity 2017;42: 801-7.

8. Oliveros H, Villamor E. Obesity and mortality in critically ill adults: a systematic review and meta-analysis. Obesity (Silver Spring) 2008;16:515-21.

9. Zhao Y, Li Z, Yang T, Wang M, Xi X. Is body mass index associated with outcomes of mechanically ventilated adult patients in intensive critical units? A systematic review and meta-analysis. PLoS One 2018;13:e0198669.

10. Singer M, Deutschman CS, Seymour CW, et al. The Third International Consensus Definitions for Sepsis and Septic Shock (Sepsis-3). JAMA 2016;315:801-10.

11. Soto GJ, Frank AJ, Christiani DC, Gong MN. Body mass index and acute kidney injury in the acute respiratory distress syndrome. Critical Care Medicine 2012;40:2601-8.

12. Hainer V, Aldhoon-Hainerova I. Obesity Paradox Does Exist. Diabetes Care 2013;36:S276-S81.

13. Lasocki S. The True Obesity Paradox. Critical Care Medicine 2015;43:240-1.

14. Pepper DJ, Demirkale CY, Sun J, et al. Does Obesity Protect Against Death in Sepsis? A Retrospective Cohort Study of 55,038 Adult Patients. Crit Care Med 2019;47:643-50.

15. Pepper DJ, Sun J, Welsh J, Cui X, Suffredini AF, Eichacker PQ. Increased body mass index and adjusted mortality in
ICU patients with sepsis or septic shock: a systematic review and meta-analysis. Critical Care 2016;20.

16. Wang S, Liu X, Chen Q, Liu C, Huang C, Fang X. The role of increased body mass index in outcomes of sepsis: a systematic review and meta-analysis. BMC Anesthesiology 2017;17.

17. Tafelski S, Yi H, Ismaeel F, Krannich A, Spies C, Nachtigall I. Obesity in critically ill patients is associated with increased need of mechanical ventilation but not with mortality. Journal of Infection and Public Health 2016;9:577-85.

18. Trivedi V, Bavishi C, Jean R. Impact of obesity on sepsis mortality: A systematic review. Journal of Critical Care 2015; 30:518-24.

19. Danziger J, Chen KP, Lee J, et al. Obesity, Acute Kidney Injury, and Mortality in Critical Illness. Critical Care Medicine 2016;44:328-34.

20. Kok B, Karvellas CJ, Abraldes JG, et al. The impact of obesity in cirrhotic patients with septic shock: A retrospective cohort study. Liver International 2017;38:1230-41.

21. Garrouste-Orgeas M, Troché G, Azoulay E, et al. Body mass index. Intensive Care Medicine 2004;30:437-43.

22. Havens JM, Columbus AB, Seshadri AJ, et al. Malnutrition at Intensive Care Unit Admission Predicts Mortality in Emergency General Surgery Patients. Journal of Parenteral and Enteral Nutrition 2016:014860711667659.

23. Wurzinger B, Dünser MW, Wohlmuth C, et al. The association between body-mass index and patient outcome in septic shock: a retrospective cohort study. Wiener klinische Wochenschrift 2010;122:31-6.

24. Aldawood A, Arabi Y, Dabbagh O. Association of Obesity with Increased Mortality in the Critically Ill Patient. Anaesthesia and Intensive Care 2006;34:629-33. 